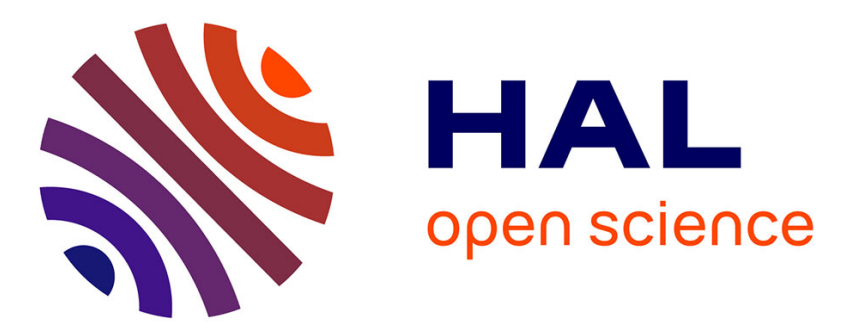

\title{
Basic reproduction ratio for a fishery model in a patchy environment
}

Pierre Auger, Ali Moussaoui, Gauthier Sallet

\section{To cite this version:}

Pierre Auger, Ali Moussaoui, Gauthier Sallet. Basic reproduction ratio for a fishery model in a patchy environment. Acta Biotheoretica, 2012, 60, pp.167-188. 10.1007/s10441-012-9155-3 . hal-01279449

\section{HAL Id: hal-01279449 \\ https://inria.hal.science/hal-01279449}

Submitted on 26 Feb 2016

HAL is a multi-disciplinary open access archive for the deposit and dissemination of scientific research documents, whether they are published or not. The documents may come from teaching and research institutions in France or abroad, or from public or private research centers.
L'archive ouverte pluridisciplinaire HAL, est destinée au dépôt et à la diffusion de documents scientifiques de niveau recherche, publiés ou non, émanant des établissements d'enseignement et de recherche français ou étrangers, des laboratoires publics ou privés. 


\title{
Basic Reproduction Ratio for a Fishery Model in a Patchy Environment.
}

\author{
P. Auger • A. Moussaoui • G. Sallet
}

Received: 15 November 2011 / Accepted: 25 February 2012

\begin{abstract}
We present a dynamical model of a multi-site fishery. The fish stock is located on a discrete set of fish habitats where it is catched by the fishing fleet. We assume that fishes remain on fishing habitats while the fishing vessels can move at a fast time scale to visit the different fishing sites. We use the existence of two time scales to reduce the dimension of the model : we build an aggregated model considering the habitat fish densities and the total fishing effort. We explore a regulation procedure, which imposes an average residence time in patches. Several equilibria exist, a Fishery Free Equilibria (FFEs) as well as a Sustainable Fishery Equilibria (SFEs). We show that the dynamics depends on a threshold which is similar to a basic reproduction ratio for the fishery. When the basic reproduction ratio is less or equal to 1 , one of the FFEs is globally asymptotically stable (GAS), otherwise one of the SFEs is GAS.
\end{abstract}

Keywords Population dynamics · stock-effort model · Time scales, · aggregation of variables $\cdot$ stability.

Pierre Auger

UMI IRD 209, UMMISCO, \& université Pierre et Marie Curie, Paris VI.

IRD France Nord, F-93143, Bondy,

France

E-mail: Pierre.Auger@ird.fr

Ali Moussaoui

Université Aboubekr Belkaid

Département de Mathématiques,Tlemcen

Algérie

E-mail: moussaouidz@yahoo.fr

Gauthier Sallet

INRIA project team : MASAIE

INRIA-Nancy Grand Est

and

UMMISCO, Université Gaston Berger, Saint-Louis

Sénégal

E-mail: Gauthier.Sallet@inria.fr 
PACS 34A34 $\cdot$ 34D23 $\cdot$ 34D40 $\cdot$ 92D30

Many mathematical models have been developed to describe the dynamics of fisheries (Smith 1968, 1969; Clark 1976, 1985, 1990). The simplest models assume a logistic equation for the fish stock with a term of catch that is proportional to the stock and to a constant fishing effort (given, for example, by the number of vessels involved in the fishery). The next step is to add an equation for the fishing effort, leading to predator-prey types of fishery models. This is the model of V.L. Smith (Smith 1968, 1969).

This paper is situated in this general context and illustrates a multi-site fishery management model. We suppose that the fish stocks distribute themselves among several patches of resources corresponding to an Ideal Free Distribution (Fretwell and Lucas 1970), i.e., fishes will aggregate in various patches proportionately to the amount of resources available in each. We study fishing activity on different spatial zones with vessel displacements occurring between the zones. Those zones or patches could be composed with some artificial reefs (Claudet and Pelletier 2004; Grossman et al. 1997) or else fish Artificial Habitats (AHs) built of different kinds of materials that have been deposited at the bottom of the sea (Rooker et al. 1997; Bonsack et al. 1991). Such AHs allow some fish species to live there and find some protection against predators. As a consequence, we consider a commercial species which is colonizing the patches (AHs) and is rather sedentary such that we can assume that it does not move from patch to patch. Furthermore, we assume that the fishing zones are close enough for the displacement of vessels to take place at a faster time scale than local interactions within the fishing zones. We assume that the displacement rates of vessels between zones is constant such as in (Auger et al. 2010).

The rationale for this hypothesis is that we look for management rules for the multi-site fishery.

A first possibility would be to avoid any regulation and to let the fishermen visit and catch fishes in the different patches as they wish. In that case, fishermen would probably visit patches and stay on a patch when the resource is important, otherwise leave that patch. In this eventuality the vessels displacement should be fish density dependent.

The second possibility is that the government or a a Fishery Institute impose some regulations. For example, the government can lay down average residence time in a given patch. According to the kind of AHs, and on the basis of the ideal free distribution (Fretwell and Lucas 1970), the different patches are supposed to attract more or less fishes. Therefore patches are characterized by different carrying capacities.

We shall look for choices of constant displacement rates of vessels that must be chosen in order to maintain a global multi-site persistent fishery. A simple situation of 3 patches has been investigated in this spirit (Mchich et al. 2006). In this reference, it has been shown that the global fishing effort can be maximized for an adequate distribution of the fishing effort.

In this paper we provide an example of choice of constant rates of boat displacement that could be made. It is not obvious to determine the carrying capacity of an AH (Grossman et al. 1997). However, It would make sense to 
think that carrying capacities of AHs depend on the size of the patch. A first possibility would then be to impose a kind of Ideal Free Distribution (IFD) for the fishing fleet (Fretwell and Lucas 1970). For example, let us consider a simple 1D chain of patches, each patch $i$ being simply connected to two neighbouring patches, $i-1$ and $i+1$. The fishery institute can decide to impose that the fishing fleet is spatially distributed in the same proportions as assumed patch carrying capacities. In our model, this can be achieved by imposing that the displacement rates of boats are constant and inversely proportional with respect to the carrying capacity of a patch. In this way, boats would distribute according to proportions similar to carrying capacities of the sites. It is well known that animals frequently distribute between different patches according to the resource available locally, using IFD. It would thus make sense that the fishery institute would recommend to the fishing fleet to distribute according to IFD. This would allow to maintain a global persistent multi-site fishery which will be investigated later on, using the reduced aggregated model.

The manuscript is organized as follows. In the next section we present a complete model which consists in a system of $2 N$ ordinary differential equations, governing the evolution of the stock in the $N$ patches and the $N$ fishing efforts on each patch.

The model includes two time scales, a fast one associated to the movements of boats between patches, and a slow one depending from the growth of the fish population and from the variation of the total number of vessels involved in the fisheries.

We take advantage of these two time scales to build a reduced model. To achieve the reduction, we apply aggregation methods of variables (Iwasa et al. 1987, 1989; Auger et al. 2008a,b), which are based on perturbation technics and on the application of an adequate version of the Center Manifold Theorem. The reduced model, called aggregated model, describes the dynamics of the total fish stock and the total fishing effort. Then, in section 3, we obtain a reduced model and consider the different equilibria. We defined a threshold for this system, which is similar to a basic reproduction ratio in epidemiology models. When this fishery reproduction ratio is smaller than one, the fishery cannot persist and the global fishing effort vanishes. Otherwise, there exists an equilibrium for a sustainable fishery that is globally asymptotically stable. Section 4 is devoted to the proof of the global stability of the fishery-extinction equilibrium when the threshold is less or equal to 1 . Section 5 proves the global stability of the fishery's viable equilibrium, i.e., this equilibrium corresponds to a sustainable fishing activities. In section 6 , we consider a two patches example and some numerical examples are taken to illustrate the results and in section 7 , we consider how our results can be used to optimize a fishery with a given set of fishing sites. 
1 The complete model

We consider $N$ patches : a patch is a geographical zone where a fish population is located. We assume that the fish population stays in its patch. This is sensible either if the patch is sufficiently large or in the case of artificial habitats (AHs) (Dempster and Taquet 2004; Moreno et al. 2007). This a discrete geographic model, since the patches are separated. This corresponds to a metapopulation model (Hanski and Gilpin 1997; Hanski 1999; Ovaskainen and Hanski 2001). The fishing boats are moving between patches. Since the boat movements are in order of hours or days and the dynamics of the fish populations and the dynamics of the fishing fleet are in order of years, it is sensible to assume that the boats are moving at a fast time scale $t=\varepsilon \tau$ where $t$ is the slow time scale and $\varepsilon$ a small dimensionless parameter.

The boats move from one patch to another according sea routes. Then the patches and the routes between patches form a digraph. The vertex of the digraph are the patches and the edges are the routes between the patches, as in figure 1 . Then we can naturally associate a digraph to a fishery. We do not assume that this digraph is symmetric. We only assume that this digraph is strongly connected. A strongly connected digraph is one in which any vertex can be reached from any other vertex by a path. This assumption is natural for a fishing fleet and a fish campaign. This is equivalent to say that the fleet starting from an entry point, is visiting all the patches and quit by the entry point. It is well known that a digraph is strongly connected if, and only if it has a strong Hamiltonian walk (Bang-Jensen and Gutin 2009). Since a general digraph can be decomposed in the sum of strongly connected components, our result permits to consider a general situation by studying each strong component of the fishery's digraph. 


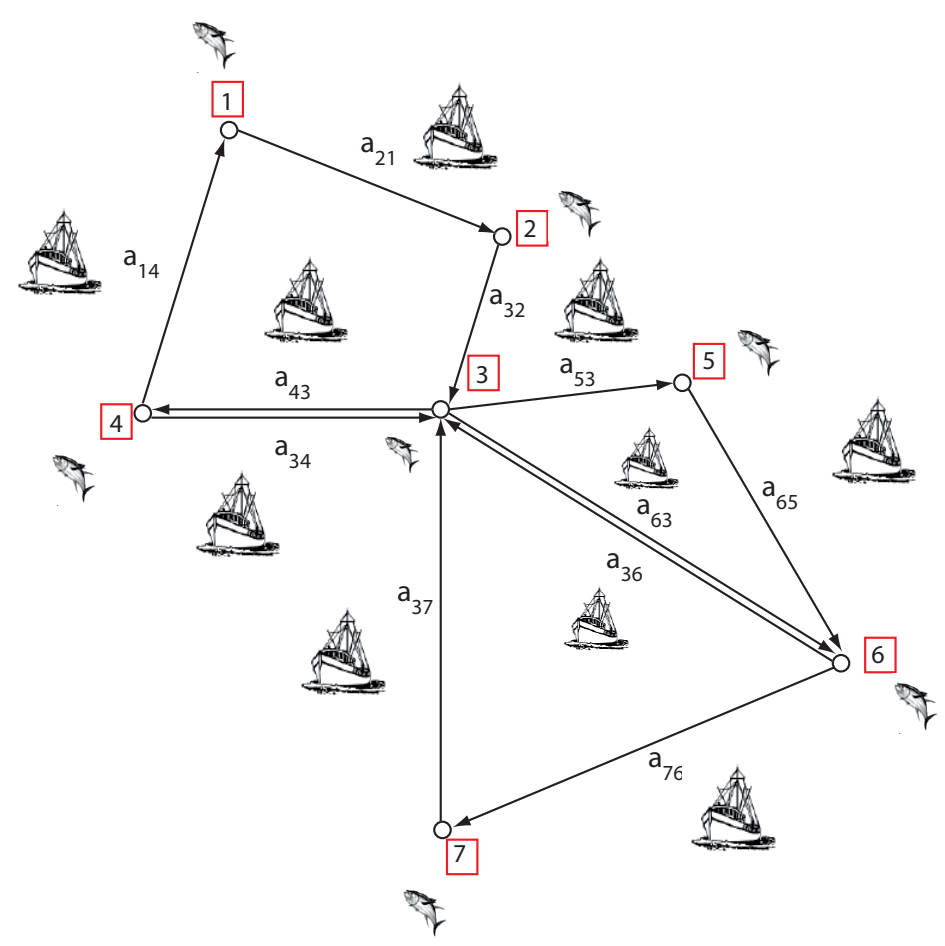

Fig. 1 Example of fishery with a strongly connected associated digraph

We assume that the moving rate between two patch is constant. Then we associate to each edge a label (i.e., a weight) $a_{i j}$ which is the rate of moving from the vertex $j$ to vertex $i$. For an example see figure 1 . Note the order of the subscripts, which is chosen for matrix multiplication reasons. Then we have a labelled digraph, which is also known as a Coates digraph (de Camino-Beck et al. 2009; Coates 1959; Doob 1984). To this Coates digraph is associated the matrix $A$. The correspondence of a digraph with the coefficient matrix of a system of equations has been pioneered in the engineering literature (Coates 1959; Mason and Zimmermann 1960). See (Chen 1976) chapter 3, for a detailed description and further references. For a given nonnegative matrix $A$ the digraph $G(A)$, described above, is the Coates digraph of $A$. Note that, given a graph $G(A)$, the matrix $A$ is the transpose of that often used as the weighted adjacency matrix; see, e.g., (Harary 1962)

We denote by $\mathbf{x} \in \mathbb{R}^{N}$ the vector whose components $\mathbf{x}_{i}$ are the fish spatial density in patch $i$. Similarly let $\mathbf{E}$ be the vector the vector whose components $E_{i}$ are the fishing effort in each patch.

The dynamics is now classically (Smith 1968, 1969; Mchich et al. 2002), described by 


$$
\left\{\begin{array}{l}
\dot{\mathbf{x}}_{i}=\varepsilon\left[r_{i} \mathbf{x}_{i}\left(1-\frac{\mathbf{x}_{i}}{K_{i}}\right)-q_{i} \mathbf{x}_{i} E_{i}\right] \\
\dot{\mathbf{E}}_{i}=\sum_{j \neq i} a_{i j} E_{j}-\sum_{j \neq i} a_{j i} E_{i}+\varepsilon\left(p_{i} q_{i} \mathbf{x}_{i}-c_{i}\right) E_{i}
\end{array}\right.
$$

Where the upper dot denotes the derivatives with respect to $\tau$. The population dynamics of fishes in patch $i$ is given a Pearl-Verhulst logistic equation with $r_{i}$ the constant growth rate and $K_{i}$ the carrying capacity. The parameter $q_{i}$ is the catchability coefficient of the fish. The parameter $p_{i}$ is the price per unit of fish and $c_{i}$ represents the cost per unit of fishing effort.

Hereafter we denote $x \leq y$ if, for any subscript $i, x_{i} \leq y_{i} ; x<y$ if $x \leq y$ and $x \neq y ; x \ll y$ if $x_{i}<y_{i}$ for any subscript $i$;

Similarly to the vector $\mathbf{x}$ we define the positive vectors of $\mathbb{R}_{+}^{N}: \mathbf{r} \gg 0, \mathbf{K} \gg 0$, $\mathbf{q} \gg 0, \mathbf{p} \gg 0$ and $\mathbf{c} \gg 0$.

If $X$ is a vector, with either $X \in \mathbb{R}^{p}$ we denote by $\operatorname{diag}(X)$ the $p \times p$ diagonal matrix whose diagonal is given by the components of $X$ and the other entries are zero.

The coefficient of the $N \times N$ matrix $A$ are defined by $a_{i j}$ for $i \neq j$ and $a_{i i}=-\sum_{j \neq i} a_{j i}$.

It is clear that $A$ is a compartmental matrix (Jacquez and Simon 1993), with zero column sum. In other words if we denote by $\mathbb{1}$ the vector of $\mathbb{R}^{N}$, $(1,1, \cdots, 1)^{T}$ we have

$$
A^{T} \mathbb{1}=0 .
$$

Now, with these notations, the system (1) can be written "vectorially"

$$
\left\{\begin{array}{l}
\dot{\mathbf{x}}=\varepsilon\left[\operatorname{diag}(\mathbf{r}) \operatorname{diag}\left(\mathbb{1}-\operatorname{diag}(\mathbf{K})^{-1} \mathbf{x}\right)-\operatorname{diag}(\mathbf{q}) \operatorname{diag}(\mathbf{E})\right] \mathbf{x} \\
\dot{\mathbf{E}}=[A+\varepsilon(\operatorname{diag}(\mathbf{p}) \operatorname{diag}(\mathbf{q}) \operatorname{diag}(\mathbf{x})-\operatorname{diag}(\mathbf{c}))] \mathbf{E}
\end{array}\right.
$$

It is straightforward to check that the nonnegative orthant $\mathbb{R}_{+}^{2 N}$ is positively invariant by system (2). This comes from the fact that the matrices appearing in (2) are Metzler matrices (Luenberger 1979; Jacquez and Simon 1993), which are known to let the nonnegative orthant positively invariant. The system is well posed. 


\section{Aggregated model}

\subsection{Fast equilibria}

The fast system is defined when $\varepsilon=0$, in other words by $\dot{\mathbf{x}}=0$ and $\dot{\mathbf{E}}=A \mathbf{E}$ The sum of the fishing effort is $\mathcal{F}=\langle\mathbf{E} \mid \mathbb{1}\rangle$, where $\langle\mid\rangle$ denotes the standard inner product of $\mathbb{R}^{N}$. For the fast system, the total fishing effort is constant:

$$
\dot{\mathcal{F}}=\langle\dot{\mathbf{E}} \mid \mathbb{1}\rangle=\langle A \mathbf{E} \mid \mathbb{1}\rangle=\left\langle\mathbf{E} \mid A^{T} \mathbb{1}\right\rangle=0 .
$$

The matrix $A$ is an irreducible matrix (Berman and Plemmons 1994), since the graph $G(A)$ is strongly connected. The matrix $A$ is a Metzler matrix (Luenberger 1979; Jacquez and Simon 1993). The stability modulus $s(M)$ of a matrix $M$ is the largest real part of the elements of the spectrum $\operatorname{Spec}(M)$ of $M$.

$$
s(M)=\max _{\lambda \in \operatorname{Spec}(M)} \operatorname{Re}(\lambda) .
$$

If $A$ is an irreducible Metzler matrix, $A^{T}$ also.

It is an immediate consequence of the Perron-Frobenius theorem (Luenberger 1979; Berman and Plemmons 1994; Smith 1995) that there exists an unique positive vector $w \gg 0$, with $\langle w \mid \mathbb{1}\rangle=1$, such that

$A^{T} w=s\left(A^{T}\right) w$. By Gersghorin theorem, the spectrum of $A$ is contained in the closed left half plane. Since $A^{T} \mathbb{1}=0$, this proves that $s(A)=0$. By irreducibility this eigenvalue is simple (Smith 1995). Then all the other eigenvalues are in the left open half plane.

This proves that any affine hyperplane $\mathcal{F}=k$, is invariant by the fast system $\dot{\mathbf{E}}=A \mathbf{E}$, and that on this affine hyperplane the system is globally asymptotically stable. Since $A$ is Metzler irreducible, using the converse of PerronFrobenius, we know that there exists an unique vector $v \gg 0$, with $\langle v \mid \mathbb{1}\rangle=1$ such that $A v=0$. The equilibrium in the manifold $\{\mathcal{F}=k\}$ is simply given by $k v=\mathcal{F} v$.

Then we have proven that the equilibrium for the fast system is given by $E_{i}=\mathcal{F} v_{i}$, where $v$ is the unique vector defined above.

In the sequel, this unique vector $v$ will be designated as the normalized Perron-Frobenius eigenvector of the Metzler matrix $A$.

For future reference, this vector $v$ is uniquely defined, from the Metzler matrix $A$ defined by the Coates graph, by the relations

$$
A v=0 \quad \text { and } \quad \sum_{i}^{n} v_{i}=1
$$

\subsection{Aggregated Model}

Aggregation methods take advantage of two time scales to derive, from a complete model, a reduced model that describes the time evolution of global 
variables at the slow time scale. Here the global variables are the local fish densities on each patches and the total fishing effort obtained along the patches. For aggregation methods we refer to (Michalski et al. 1997).

The aggregated model is obtained by making an approximation. This approximation consists in assuming that the fast variables are at the fast equilibrium. The dynamic behavior of the reduced system provides a good approximation of the dynamic behavior of the complete system, when the "parameter" $\varepsilon$ is small enough and when the aggregated model is structurally stable (Auger et al. 2008b; Iwasa et al. 1989). The aggregated model is obtained by substituting the fast and stable equilibrium into the complete system with the equation of the total fishing effort. This leads to the following structurally stable model:

$$
\left\{\begin{array}{l}
\dot{\mathbf{x}}_{i}=r_{i} \mathbf{x}_{i}\left(1-\frac{\mathbf{x}_{i}}{K_{i}}\right)-q_{i} \mathbf{x}_{i} v_{i} \mathcal{F} \\
\dot{\mathcal{F}}=\sum_{i=1}^{N}\left(p_{i} q_{i} \mathbf{x}_{i}-c_{i}\right) v_{i} \mathcal{F} .
\end{array}\right.
$$

Or equivalently

$$
\left\{\begin{array}{l}
\dot{\mathbf{x}}_{i}=r_{i} \mathbf{x}_{i}\left(1-\frac{\mathbf{x}_{i}}{K_{i}}\right)-a_{i} \mathbf{x}_{i} \mathcal{F} \\
\dot{\mathcal{F}}=\left(\sum_{i=1}^{N} p_{i} a_{i} \mathbf{x}_{i}-c\right) \mathcal{F}
\end{array}\right.
$$

with $a_{i}=q_{i} v_{i}$ and $c=\sum_{i} c_{i} v_{i}$. Since $v \gg 0, \mathbf{q} \gg 0$ and $\mathbf{c} \gg 0$, we note that $\mathbf{a} \gg 0$ and $c>0$.

The parameter $a_{i}$ captures the catchability in the fishes in patch $i$ and the influence of the Coates graph, since $v_{i}$ is the $i$-th component of the PerronFrobenius eigenvector of $A$, which depends only of the structure of the graph (i.e., the edges and the weights of the edges).

For this system the nonnegative orthant is clearly positively invariant. This system can be interpreted as a Lotka-Volterra system with preys on distinct patches and a "predator" (here $\mathcal{F}$ ) feeding on the different patches.

The faces (see definition 2.14 of (Berman and Plemmons 1994)) of $\mathbb{R}_{+}^{N+1}$ are positively invariant. The faces of $\mathbb{R}_{+}^{N+1}$ are of the form

$$
\mathcal{H}_{J}=\left\{z \in \mathbb{R}_{+}^{N+1} \mid z_{i}=0 \text { if } i \notin J\right\} .
$$

The singleton $\{0\}$ and the nonnegative orthant are also faces of the nonnegative orthant.

There exists a "extinction" equilibrium given by $\mathbf{x}=0$ and $\mathcal{F}=0$, where there is no fishes and no fishing. There exists "predator-free" equilibrium in 
the positive orthant given by $\mathbf{x}=\mathbf{K}$ and $\mathcal{F}=0$. By analogy with the epidemiological situation, we will call this equilibrium a "Fishing Free Equilibrium" (FFE).

In each interior of a face $\mathcal{H}_{J}$ there exists a FFE given by $\mathcal{F}=0$ and $x_{i}=K_{i}$ for $i \in J$ and $x_{i}=0$ otherwise. We have $\left(2^{N}-1\right)$ fishing free equilibria.

We claim that the "extinction" equilibrium is always unstable.It suffices to consider the Jacobian matrix computed at this equilibrium, since the $r_{i}>0$ are eigenvalues of this matrix.

In a completely analogous way, as in epidemiology, we can define the basic reproduction ratio of the "predator" (Diekmann et al. 1990; Diekmann and Heesterbeek 2000). The concept of basic reproduction ratio originates in demography. It was Dublin and Lotka who formalized the computation and introduced the notation $\mathcal{R}_{0}$ (Heesterbeek 2002).

This is the expected number of secondary predator produced by a typical predator, during its total life, when introduced in a population of preys (at equilibrium) without predator. In our context the basic reproduction ratio for a fishery will be the expected generated fishing effort (i.e., in an economic sense) produced by a given fishing effort, during its mean time duration, when applied to a new population of non fished fish, at demographic equilibrium. From equation (5), the mean duration time of the economic fishing effort, is $\frac{1}{c}$, while the secondary product, by unit of time, is $\sum_{i=1}^{N} p_{i} a_{i} \mathbf{K}_{i}$, when at the carrying capacity.

It is not difficult to be convinced, using for example the approach of (van den Driessche and Watmough 2002), that

$$
\mathcal{R}_{0}=\frac{\sum_{i=1}^{N} p_{i} a_{i} K_{i}}{c} .
$$

Independently of any interpretation, $\mathcal{R}_{0}$ is simply a convenient threshold for bifurcation behaviour.

Using the results of (Diekmann et al. 1990) we know that $\mathcal{R}_{0}$ is a threshold and that if $\mathcal{R}_{0}<1$ then the fishing-free equilibrium (FFE) is locally asymptotically stable, and if $\mathcal{R}_{0}>1$ this equilibrium is unstable.

We are now, looking for other equilibria, for which $\mathcal{F}^{\star}>0$, i.e., equilibria for which the fishing effort is non zero. For these equilibria, the fishing effort is sustainable and the fishery is economically viable. We will call these equilibria Sustainable Fishing Equilibria (SFE). We denote by $\left(\mathbf{x}^{\star}, \mathcal{F}^{\star}\right)$ such an equilibrium, with $\mathcal{F}^{\star} \neq 0$. Let $I$ be the set of subscripts such that $\mathbf{x}_{i}^{\star}=0$, and the $J$ the complement of $I$ in $[1, \cdots, N]$. We consider the face $\mathcal{F}_{J}$.

We have, for $i \in J$ the relation

$$
\mathbf{x}_{i}^{\star}=K_{i}\left(1-\frac{a_{i}}{r_{i}} \mathcal{F}^{\star}\right) \quad \text { for } i \in J
$$

We deduce 


$$
\mathcal{F}_{J}^{\star}=\frac{\frac{1}{c} \sum_{i \in J} p_{i} a_{i} K_{i}-1}{\frac{1}{c} \sum_{i \in J} \frac{p_{i} a_{i}^{2} K_{i}}{r_{i}}} .
$$

We use the subscript $J$ to stress the relation with the face $\mathcal{H}_{J}$. Analogously we define, for the face $\mathcal{H}_{J}$

$$
\mathcal{R}_{0, J}=\frac{1}{c} \sum_{i \in J} p_{i} a_{i} K_{i}
$$

We have

$$
0<\mathcal{F}_{J}^{\star} \leq \frac{\mathcal{R}_{0}-1}{\frac{1}{c} \sum_{i \in J} \frac{p_{i} a_{i}^{2} K_{i}}{r_{i}}} .
$$

Hence necessary conditions for existence of an equilibrium with sustainability of the fishing effort are $\mathcal{R}_{0}>1$ and $\mathcal{R}_{0, J}>1$. Since $\mathbf{p} \gg 0$ and $\mathbf{a} \gg 0$, we have clearly $\mathcal{R}_{0}>\mathcal{R}_{0, J}$. Moreover the equilibria has a biological meaning if it is contained in the nonnegative orthant, then we must have the inequality

$$
\mathcal{F}_{J}^{\star}<\min _{i \in J} \frac{r_{i}}{a_{i}} .
$$

Eventually by reordering the coordinates, we can assume that we have the following inequalities

$$
\frac{r_{1}}{a_{1}} \leq \frac{r_{2}}{a_{2}} \leq \cdots \leq \frac{r_{N}}{a_{N}} .
$$

In other words, we renumber the patches, according this criteria, which involves the growth rate of the patch $a_{i}$, its catchability $q_{i}$ and $v_{i}$ the component of the Perron-Frobenius eigenvector of the associated Coates graph.

We will adopt this ordering in the sequel.

If $J=\left\{i_{1} \leq i_{2} \leq \cdots \leq i_{k}\right\}$ then inequality (8) reads

$$
\mathcal{F}_{J}^{\star}=\frac{\sum_{i \in J} p_{i} a_{i} K_{i}-c}{\sum_{i \in J} \frac{p_{i} a_{i}^{2} K_{i}}{r_{i}}} \leq \frac{r_{i_{1}}}{a_{i_{1}}} .
$$

equivalently 


$$
\frac{p_{i_{1}} a_{i_{1}} K_{i_{1}}+\sum_{\substack{i \in J \\ i>i_{1}}} p_{i} a_{i} K_{i}-c}{\frac{p_{i_{1}} a_{i_{1}}^{2} K_{i_{1}}}{r_{i_{1}}}+\sum_{\substack{i \in J \\ i>i_{1}}} \frac{p_{i} a_{i}^{2} K_{i}}{r_{i}}}<\frac{r_{i_{1}}}{a_{i_{1}}} .
$$

Then we have

$$
p_{i_{1}} a_{i_{1}} K_{i_{1}}+\sum_{\substack{i \in J \\ i>i_{1}}} p_{i} a_{i} K_{i}-c<p_{i_{1}} a_{i_{1}} K_{i_{1}}+\sum_{\substack{i \in J \\ i>i_{1}}} p_{i} a_{i} K_{i} \frac{r_{i_{1}} a_{i}}{a_{i_{1}} r_{i}}
$$

Finally the condition is

$$
c>\sum_{\substack{i \in J \\ i>i_{1}}} p_{i} a_{i} K_{i}\left(1-\frac{r_{i_{1}}}{a_{i_{1}}} \frac{a_{i}}{r_{i}}\right) .
$$

The fishery has a SFE if $\mathcal{R}_{0, J}>1$ and if there exits a subset of subscripts $J \subset[1, \cdots, N]$ such that the inequality (11) is satisfied. This equilibrium is in the interior of the $\mathcal{H}_{J}$ face. We can have again $\left(2^{N}-1\right)$ sustainable fishing equilibria.

When $J=[1, \cdots, N]$ then the face is the nonnegative orthant.

To summarize a SFE exists, if it exists a subset of subscripts $J$ such that

$$
\sum_{i \in J} p_{i} a_{i} K_{i}>c>\sum_{\substack{i \in J \\ i>i_{1}}} p_{i} a_{i} K_{i}\left(1-\theta_{i}\right)
$$

Where $\theta_{i}=\frac{r_{i_{1}}}{a_{i_{1}}} \frac{a_{i}}{r_{i}} \leq 1$ and $J=\left\{i_{1} \leq i_{2} \leq \cdots \leq i_{N}\right\}$.

Conclusion : we have found in each face, excepted the face $\{0\}$, a FFE equilibrium and the possibility of a SFE. The equilibria in the proper faces can also, by analogy with metapopulation models, be called "mixed equilibrium" (Arino 2009). Finally we found that there are $2^{N+1}-1$ possible equilibria. The SFE in the positive orthant is the most interesting, from the fishery point of view.

\section{Global stability of the "fishery-free" equilibrium}

We have the following result

\section{Theorem 1}

The equilibrium $(\mathbf{K}, 0) \in \mathbb{R}_{+}^{N+1}$ is globally asymptotically stable on the positive orthant for system (4) iff $\mathcal{R}_{0} \leq 1$. 
A FFE which is in a face $\mathcal{H}_{J}$ is globally asymptotically stable in the interior of this face iff

$$
\mathcal{R}_{0, J}=\frac{\sum_{i \in J} p_{i} a_{i} K_{i}}{c} \leq 1 .
$$

\section{Proof}

For the first assertion, the condition is necessary since this equilibrium is unstable if $\mathcal{R}_{0}>1$.

We assume $\mathcal{R}_{0} \leq 1$ and we consider the following Lyapunov-Volterra function

$$
V(\mathbf{x}, \mathcal{F})=\sum_{i=1}^{N} p_{i}\left(\mathbf{x}_{i}-K_{i} \ln \mathbf{x}_{i}-K_{i}+K_{i} \ln K_{i}\right)+\mathcal{F} .
$$

defined on $\mathbb{R}_{*+}^{N} \times \mathbb{R}_{+}$. This function has a long history of application to LotkaVolterra models (Beretta and Takeuchi 1988; Goh 1977; Harrison 1979; Takeuchi and Adachi 1980; Hofbauer and Sigmund 1998) and was originally discovered by Volterra himself (Volterra 1931) p.15, although he did not use the vocabulary and the theory of Lyapunov functions.

This domain is positively invariant by system (4) and contains $(\mathbf{K}, 0)$. The function $V$ is positive definite on the domain and radially unbounded (Hale 1980).

The derivative of $V$ along the trajectories is given by

$$
\begin{aligned}
\dot{V} & =\sum_{i=1}^{N} p_{i} \frac{\mathbf{x}_{i}-K_{i}}{\mathbf{x}_{i}} \dot{\mathbf{x}}_{i}+\dot{\mathcal{F}} \\
& =-\sum_{i=1}^{N} p_{i} r_{i} \frac{\left(\mathbf{x}_{i}-K_{i}\right)^{2}}{K_{i}}-\sum_{i=1}^{N} p_{i} a_{i}\left(\mathbf{x}_{i}-K_{i}\right) \mathcal{F}+\sum_{i=1}^{N} p_{i} a_{i} \mathbf{x}_{i} \mathcal{F}-c \mathcal{F} \\
& =-\sum_{i=1}^{N} p_{i} r_{i} \frac{\left(\mathbf{x}_{i}-K_{i}\right)^{2}}{K_{i}}+\left(\sum_{i=1}^{N} p_{i} a_{i} K_{i}-c\right) \mathcal{F} \\
& =-\sum_{i=1}^{N} p_{i} r_{i} \frac{\left(\mathbf{x}_{i}-K_{i}\right)^{2}}{K_{i}}+\frac{1}{c}\left(\mathcal{R}_{0}-1\right) \mathcal{F} \leq 0 .
\end{aligned}
$$

When $\mathcal{R}_{0}<1$ this function negative definite, when $\mathcal{R}_{0}=1$ we conclude by using, classically, the LaSalle's invariance principle (LaSalle 1976; Hale 1980). Since $V$ is radially unbounded the stability is global on the domain.

The proof of the second assertion is completely similar to the preceding proof, and it suffices to restrain to the face $\mathcal{F}_{J}$, so we omit it.

\section{Corollary 1}

A necessary condition for the viability of the fishery is $\mathcal{R}_{0}>1$. 


\section{Global Stability of the SFEs}

In this section we will assume that $\mathcal{R}_{0}>1$. We will completely describe, in this case, the stability analysis of system (4).

We consider a subset $I \subset[1, \cdots, N]$ and $J$ its complement in $[1, \cdots, N]$ for which the condition (8) is satisfied. The SFE is defined by the relations (6) and (7). This equilibrium belongs to the positively invariant face (Berman and Plemmons 1994) $\mathcal{H}_{J}$ of the nonnegative orthant $\mathbb{R}^{N+1}$, defined by

$$
\mathcal{H}_{J}=\left\{(\mathbf{x}, \mathcal{F}) \in \mathbb{R}_{+}^{N+1} \mid \mathbf{x}_{i}=0 \text { for } i \in I\right\} .
$$

Equivalently the face $\mathcal{H}_{J}$ is the positive cone generated by the vector $e_{i}$ of the canonical basis of $\mathbb{R}^{N}$, for $i \in J$, where $J$ is the complement of $I$.

Our first result will give the stability analysis in a face.

\section{Theorem 2}

If a SFE exists, then it is globally asymptotically stable in the interior of its face $\mathcal{H}_{J}$ for system (4).

\section{Remark 1}

If a viable equilibrium $\left(\mathrm{x}^{\star}, \mathcal{F}^{\star}\right)$ exists in the interior of the nonnegative orthant, i.e., $\mathbf{x}^{\star} \gg 0$ and $\mathcal{F}^{\star}>0$, this equilibrium is globally asymptotically stable in the positive orthant.

\section{Proof of the theorem}

For the simplicity of the exposition we assume $J=[1, \cdots, N]$. Adapting the proof for a SFE in a face is straightforward.

We consider the following Lyapunov function

$V(\mathbf{x}, \mathcal{F})=\sum_{i=1}^{N} p_{i}\left(\mathbf{x}_{i}-\mathbf{x}_{i}^{\star}-\mathbf{x}_{i}^{\star} \ln \mathbf{x}_{i}+\mathbf{x}_{i}^{\star} \ln \mathbf{x}_{i}^{\star}\right)+\left(\mathcal{F}-\mathcal{F}^{\star} \ln \mathcal{F}-\mathcal{F}^{\star}+\mathcal{F}^{\star} \ln \mathcal{F}^{\star}\right)$.

This function is defined in the interior of the nonnegative orthant, positive definite and radially unbounded (Hale 1980). Note that the positive definiteness is a consequence of the positivity of the $\mathbf{x}_{i}^{\star}$ and $\mathcal{F}^{\star}$.

For the ease of computation we rewrite system (4):

$$
\left\{\begin{array}{l}
\left.\dot{\mathbf{x}}_{i}=\left[\frac{r_{i}}{a_{i}}\left(1-\frac{\mathbf{x}_{i}}{K_{i}}\right)-\frac{r_{i}}{a_{i}}\left(1-\frac{\mathbf{x}_{i}^{\star}}{K_{i}}\right)-\mathcal{F}+\mathcal{F}^{\star}\right)\right] a_{i} \mathbf{x}_{i} \\
\dot{\mathcal{F}}=\left(\sum_{i=1}^{N} p_{i} a_{i}\left(\mathbf{x}_{i}-\mathbf{x}_{i}^{\star}\right)\right) \mathcal{F}
\end{array}\right.
$$

More simply 


$$
\left\{\begin{array}{l}
\left.\dot{\mathbf{x}}_{i}=\left[\frac{r_{i}}{a_{i}}\left(\frac{\mathbf{x}_{i}^{\star}-\mathbf{x}_{i}}{K_{i}}\right)-\mathcal{F}+\mathcal{F}^{\star}\right)\right] a_{i} \mathbf{x}_{i} \\
\dot{\mathcal{F}}=\left(\sum_{i=1}^{N} p_{i} a_{i}\left(\mathbf{x}_{i}-\mathbf{x}_{i}^{\star}\right)\right) \mathcal{F} .
\end{array}\right.
$$

The derivative of $V$ along of the trajectories of (14) is given by

$$
\begin{aligned}
\dot{V} & =\sum_{i=1}^{N} p_{i} \frac{\mathbf{x}_{i}-\mathbf{x}_{i}^{\star}}{\mathbf{x}_{i}} \dot{\mathbf{x}}_{i}+\frac{\mathcal{F}-\mathcal{F}^{\star}}{\mathcal{F}} \dot{\mathcal{F}}, \\
& =-\sum_{i=1}^{N} \frac{p_{i} r_{i}}{K_{i}}\left(\mathbf{x}_{i}-\mathbf{x}_{i}^{\star}\right)^{2}-\sum_{i=1}^{N} p_{i} a_{i}\left(\mathbf{x}_{i}-\mathbf{x}_{i}^{\star}\right)\left(\mathcal{F}-\mathcal{F}^{\star}\right) \\
& +\sum_{i=1}^{N} p_{i} a_{i}\left(\mathbf{x}_{i}-\mathbf{x}_{i}^{\star}\right)\left(\mathcal{F}-\mathcal{F}^{\star}\right), \\
& =-\sum_{i=1}^{N} p_{i} r_{i} \frac{\left(\mathbf{x}_{i}-\mathbf{x}_{i}^{\star}\right)^{2}}{K_{i}} \leq 0 .
\end{aligned}
$$

This proves the stability of the equilibrium. The global asymptotic stability comes from Lasalle's invariance principle (Hale 1980; LaSalle 1976).

4.1 Stability analysis when $\mathcal{R}_{0}>1$.

We will prove a result of global stability in the positive orthant.

Before giving our main result, we need to precise some convenient definitions and notations. We recall that we have ordered the patches such that

$$
\frac{r_{1}}{a_{1}} \leq \frac{r_{2}}{a_{2}} \leq \cdots \leq \frac{r_{N}}{a_{N}}
$$

We will now introduce a flag manifold of faces. A flag, in a finite dimensional vector space, is an increasing sequence of subspaces (Humphreys 1975). The standard flag associated with the canonical basis is the one where the $i$-th subspace is spanned by the first $i$ vectors of the basis. Analogically we introduce the standard flag manifold of faces by defining in $\mathbb{R}_{+}^{N} \times \mathbb{R}_{+}$

$$
\mathcal{H}_{\{i, \cdots, N\}} \approx \mathbb{R}_{+}^{N-i+1} \times \mathbb{R}_{+}
$$

Then the flag is composed of the $N$ faces

$$
\mathbb{R}_{+}^{N} \times \mathbb{R}_{+}=\mathcal{H}_{\{1, \cdots, N\}} \supset \mathcal{H}_{\{2, \cdots, N\}} \supset \cdots \supset \mathcal{H}_{\{N\}} .
$$


In each face of this flag a SFE can exist. We will denote by $\left(x_{\{i, \cdots, N\}}^{\star}, \mathcal{F}_{\{i, \cdots, N\}}^{\star}\right)$ this SFE, when it exists, in the face $\mathcal{H}_{\{i, \cdots, N\}}$. We have also, in the same face, the corresponding $\operatorname{FFE}\left(0, \cdots, 0, K_{i}, K_{i+1}, \cdots, K_{n}, 0\right)$.

Concerning the SFE we have the following

\section{Proposition 1}

If $\mathcal{R}_{0}>1$ then there exists a SFE in a face $\mathcal{H}$ of the standard flag, and no $S F E$ can exist in the faces of the flag containing $\mathcal{H}$.

\section{Proof}

A SFE exists in the interior of $\mathcal{H}_{\{1, \cdots, N\}}$ if the inequality (11) is satisfied, i.e., in our case

$$
c>\sum_{i>1} p_{i} a_{i} K_{i}\left(1-\frac{r_{1}}{a_{1}} \frac{a_{i}}{r_{i}}\right) .
$$

This inequality is equivalent to

$$
r_{1}-a_{1} \mathcal{F}_{\{2, \cdots, N\}}^{\star}>0 .
$$

The existence of the SFE is then expressed with an inequality involving the an expression coming from the SFE of the next face in the flag.

We have two cases :

- Either $r_{1}-a_{1} \mathcal{F}_{\{2, \cdots, N\}}^{\star}>0$ is satisfied,

- or $r_{1}-a_{1} \mathcal{F}_{\{2, \cdots, N\}}^{\star} \leq 0$.

In the second case the inequality $r_{1}-a_{1} \mathcal{F}_{\{2, \cdots, N\}}^{\star} \leq 0$ is equivalent to

$$
\sum_{i>1} p_{i} a_{i} K_{i}\left(1-\frac{r_{1}}{a_{1}} \frac{a_{i}}{r_{i}}\right) \geq c .
$$

Since each $\left(1-\frac{r_{1}}{a_{1}} \frac{a_{i}}{r_{i}}\right)<1$, we have

$$
\mathcal{R}_{0,\{2, \cdots, N\}}=\frac{\sum_{i>1} p_{i} a_{i} K_{i}}{c}>1 .
$$

The necessary condition for existence of the equilibrium $\mathcal{F}_{\{2, \cdots, N\}}^{\star}$ in the next face $\mathcal{H}_{\{2, \cdots, N\}}$ of the flag, is satisfied. We have, again, two cases :

- Either $r_{2}-a_{2} \mathcal{F}_{\{3, \cdots, N\}}^{\star}>0$ is satisfied, and $\mathcal{F}_{\{2, \cdots, N\}}^{\star}$ exists,

- or $r_{2}-a_{2} \mathcal{F}_{\{3, \cdots, N\}}^{\star} \leq 0$ otherwise.

This is the beginning of a descending recurrence with a decision tree. We stop when we obtain a SFE, and no other SFE has been detected in the preceding faces of the flag. We have just to check that this process will finish with a SFE. In the worst case, we will arrive to the face $\mathcal{H}_{\{N\}}$ with $p_{N} a_{N} K_{N}>$ $c$. Then we get 


$$
x_{\{N\}}^{\star}=\frac{c}{p_{N} a_{N}}>0 \quad \text { and } \quad \mathcal{F}_{\{N\}}^{\star}=\frac{r_{N}}{a_{N}}\left(1-\frac{c}{p_{N} a_{N} K_{N}}\right)>0 .
$$

We will always obtain a SFE in a face of the standard flag.

This ends the proof of the proposition.

We can now state our main result

\section{Theorem 3}

When $\mathcal{R}_{0}>1$, the SFE of proposition 1 is globally asymptotically stable on the domain which is the union of the positive orthant and the interior of the face of the SFE.

Remark 2 This theorem has a biological corollary : this means that when $\mathcal{R}_{0}>1$, a fishery operating on a set of non empty patches, stabilizes to a sustainable equilibrium.

\section{Proof}

We denote by $\mathcal{F}_{\{k, \cdots, N\}}^{\star}$ ) the value of the fishing effort at the SFE obtained in proposition (1). This SFE is in the interior of the face $\mathcal{H}_{\{k, \cdots, N\}}$. Then we know that, since this SFE exists we have

$$
\frac{r_{k}}{a_{k}}>\mathcal{F}_{\{k+1, \cdots, N\}}^{\star}
$$

and moreover, from the preceding step of the recurrence

$$
\frac{r_{k-1}}{a_{k-1}} \leq \mathcal{F}_{\{k, \cdots, N\}}^{\star}
$$

We deduce immediately the following relation

$$
\frac{r_{1}}{a_{1}} \leq \frac{r_{2}}{a_{2}} \leq \cdots \leq \frac{r_{k-1}}{a_{k-1}} \leq \mathcal{F}_{\{k, \cdots, N\}}^{\star}
$$

We now consider the following Lyapunov function

$$
\begin{aligned}
V(\mathbf{x}, \mathcal{F})=\sum_{i=1}^{k-1} p_{i} \mathbf{x}_{i}+\sum_{i=k}^{N} p_{i}\left(\mathbf{x}_{i}-\mathbf{x}_{i}^{\star}-\mathbf{x}_{i}^{\star}\right. & \left.\ln \mathbf{x}_{i}+\mathbf{x}_{i}^{\star} \ln \mathbf{x}_{i}^{\star}\right) \\
& +\left(\mathcal{F}-\mathcal{F}^{\star} \ln \mathcal{F}-\mathcal{F}^{\star}+\mathcal{F}^{\star} \ln \mathcal{F}^{\star}\right) .
\end{aligned}
$$

This function is defined on the domain $\mathbb{R}_{+}^{k-1} \times \mathbb{R}_{+*}^{N-k+1} \times \mathbb{R}_{+*}$. We compute the derivative of $V$ along of the trajectories of (14).

To shorten the notation, we use for designing the components of the SFE, the notation $x_{i}^{\star}$ for $\left(\mathbf{x}_{\{k, \cdots, N\}}^{\star}\right)_{i}$ and $\mathcal{F}^{\star}$ for $\mathcal{F}_{\{k, \cdots, N\}}^{\star}$. 


$$
\begin{aligned}
& \dot{V}=\sum_{i=1}^{k-1} p_{i} r_{i} \mathbf{x}_{i}- \frac{p_{i} r_{i}}{K_{i}} \mathbf{x}_{i}^{2}-p_{i} a_{i} \mathbf{x}_{i} \mathcal{F} \\
&+\sum_{i=k}^{N}-\frac{p_{i} r_{i}}{K_{i}}\left(x-\mathbf{x}_{i}^{\star}\right)^{2}-\sum_{i=k}^{N} p_{i} a_{i}\left(\mathbf{x}_{i}-\mathbf{x}_{i}^{\star}\right)\left(\mathcal{F}-\mathcal{F}^{\star}\right) \\
&+\sum_{i=1}^{k-1} p_{i} \mathbf{x}_{i}\left(\mathcal{F}-\mathcal{F}^{\star}\right)+\sum_{i=k}^{N} p_{i} a_{i}\left(\mathbf{x}_{i}-\mathbf{x}_{i}^{\star}\right)\left(\mathcal{F}-\mathcal{F}^{\star}\right), \\
&=-\sum_{i=1}^{k-1} \frac{p_{i} r_{i}}{K_{i}} \mathbf{x}_{i}^{2}-\sum_{i=k}^{N} \frac{p_{i} r_{i}}{K_{i}}\left(\mathbf{x}_{i}-\mathbf{x}_{i}^{\star}\right)^{2}+\sum_{i=1}^{k-1} p_{i}\left(r_{i}-a_{i} \mathcal{F}^{\star}\right) \mathbf{x}_{i} .
\end{aligned}
$$

But with the inequality (16) we have for $i \leq k-1$ the relation $r_{i}-a_{i} \mathcal{F}^{\star} \leq 0$. Then $\dot{V} \leq 0$ which proves the stability of the SFE. The global stability is obtained, as usual, by Lasalle's invariance principle and the radial unboundedness of the Lyapunov function $V$.

\section{Numerical examples}

\subsection{Two patches}

When $N=2$ the reduced system is

$$
\left\{\begin{array}{l}
\dot{x}=r_{1} x\left(1-\frac{x}{K_{1}}\right)-a_{1} x \mathcal{F} \\
\dot{y}=r_{2} y\left(1-\frac{y}{K_{2}}\right)-a_{2} y \mathcal{F} \\
\dot{\mathcal{F}}=\left(-c+p_{1} a_{1} x+p_{2} a_{2} y\right) \mathcal{F} .
\end{array}\right.
$$

We can illustrate the results obtained in the preceding sections.

Assuming the ordering of coordinates $\frac{r_{1}}{a_{1}} \leq \frac{r_{2}}{a_{2}}$, we have the following bifurcation diagram, in the $\left(p_{2} a_{2} K_{2}, p_{1} a_{1} K_{1}\right)$ parameter plane, with $\theta=1-\frac{r_{1}}{a_{1}} \frac{a_{2}}{r_{2}}$. 


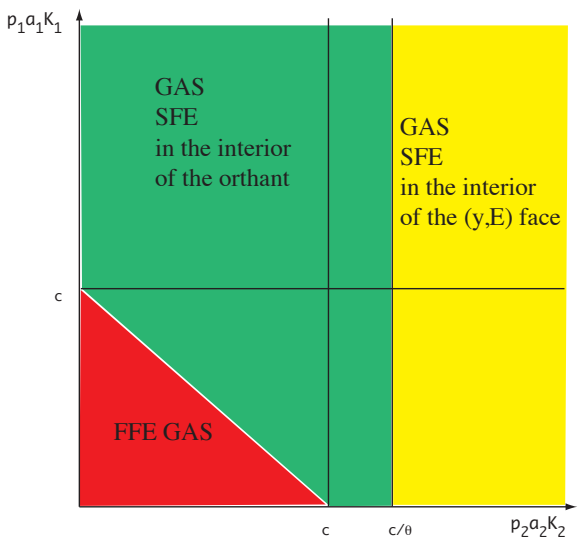

Fig. 2 Bifurcation diagram

We choose for parameters for the non-reduced system, with the notations of system (1)

Let $r_{1}=0.7, r_{2}=0.9, K_{1}=25, K_{2}=22.5, a_{12}=a_{21}=0.5, q_{1}=1, q_{2}=$ $1, c_{1}=1, c_{2}=2.2, p_{1}=0.8, p_{2}=0.5$. The rates of movement from one patch to another are equal.

With these values the vector $v$, defined by relation (3), is given by $v_{1}=v_{2}=0.5$. The others parameters are $a_{1}=a_{2}=0.5$ and $c=c_{1} v_{1}+c_{2} v_{2}=$ 1.6 .

The population densities $x, y$ and effort $\mathcal{F}$ are shown in figure 3 


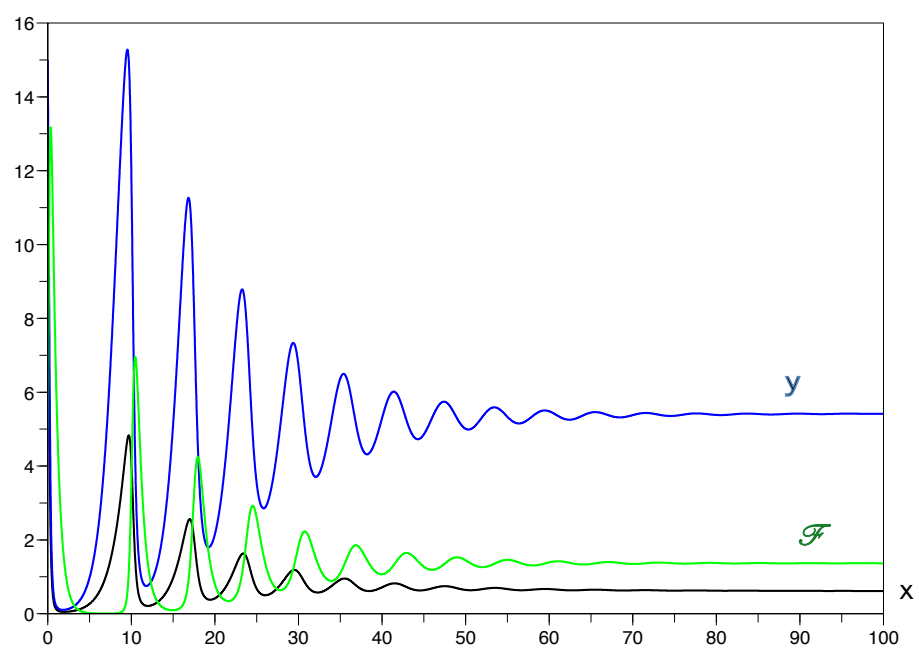

Fig. 3 Solutions curves beginning with $x_{0}=10, y_{0}=15, \mathcal{F}_{0}=5$.

We have $\mathcal{R}_{0}>1$ and condition (15) satisfied for our system, then a SFE exits in the positive orthant. The coordinates are

$$
\left[\begin{array}{l}
x^{\star} \\
y^{\star} \\
\mathcal{F}^{\star}
\end{array}\right]=\left[\begin{array}{l}
0.6086957 \\
5.426087 \\
1.365913
\end{array}\right]
$$

We obtain, by simulation on an interval of length 100 unity of of time, the final value

$(0.6144565,5.4153055,1.3652747)^{T}$.

\subsection{Example with 4 patches}

In this section we will show that, by only modifying the Coates graph of the fishery, it is possible to move a boundary SFE towards and into the positive orthant

We consider the following Coates graph of a 4 sites example 


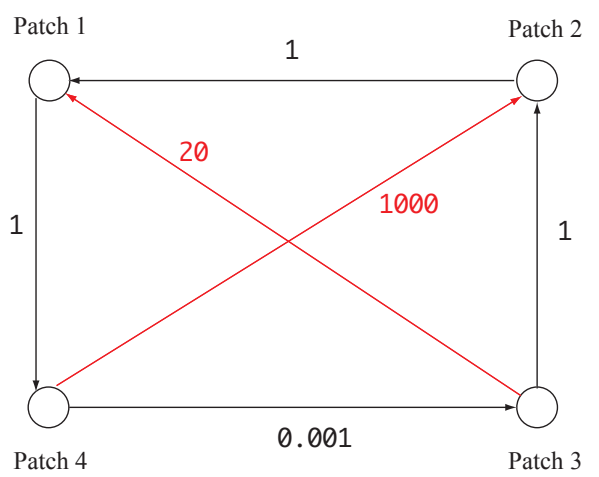

Fig. 4 Coates graph of 4 patches situtation

Using the notation of section 2.2, we choose for parameters

$$
r=\left[\begin{array}{l}
1 \\
1 \\
1 \\
1
\end{array}\right] \quad K=\left[\begin{array}{l}
1 \\
4 \\
3 \\
2
\end{array}\right] \quad q=\left[\begin{array}{l}
4 \\
1 \\
2 \\
3
\end{array}\right] \quad p=\left[\begin{array}{l}
1 \\
1 \\
1 \\
1
\end{array}\right] \quad c=\left[\begin{array}{c}
1 \\
0.1 \\
0.1 \\
6
\end{array}\right]
$$

Since we need to compute the greatest eigenvalue of a positive operator and its corresponding eigenvector we must use, in general, a numerical software. We write a simple code in Scilab ${ }^{\circledR}$ to obtain the different criteria. This code is given in appendix.

We obtain for the unique vector $v$ satisfying relation (3)

$$
v=\left[\begin{array}{c}
0.4997504 \\
0.4997499 \\
2.380 \mathrm{E}-08 \\
0.0004997
\end{array}\right]
$$

We compute the $\frac{r_{i}}{a_{i}}$ and find for $i=1, \cdots, 4$ the values

$$
\text { (0.5002498, 2.001001, 21010511., 667.00037) }
$$

We have numbered the patch such that this gives the relation (9). Then we obtain

$$
\mathcal{R}_{0}=7.2742115,
$$

but the inequality (10) is not satisfied since

$$
\mathcal{F}_{J}^{\star}=\frac{\sum_{i \in J} p_{i} a_{i} K_{i}-c}{\sum_{i \in J} \frac{p_{i} a_{i}^{2} K_{i}}{r_{i}}}=0.6908843>\frac{r_{1}}{a_{1}}=0.5002498 .
$$


Then there is no SFE in the positive orthant. We check that

$$
\mathcal{R}_{0,\{2,3,4\}}=\frac{\sum_{i=2,3,4} p_{i} a_{i} K_{i}}{c}=3.6398299>1
$$

and

$$
\mathcal{F}_{2,3,4}^{\star}=\frac{\sum_{i \in J} p_{i} a_{i} K_{i}-c}{\sum_{i \in J} \frac{p_{i} a_{i}^{2} K_{i}}{r_{i}}}=1.4534202<\frac{r_{2}}{a_{2}}=2.001001
$$

According to the proofs of section 4 there is a SFE in the face $\left(x_{2}, x_{3}, x_{4}\right)$. This SFE is globally asymptotically stable in the positive orthant and in its face.

This situation is not favorable, since this strategy exhausts patch 1 . If we suppress in the graph the red edges in figure (4),i.e., the edge from patch 4 to 2 and from patch 3 to 1 , the graph is still strongly connected and the computation gives now, new vectors $v$ and $a$ :

$$
v=\left[\begin{array}{l}
0.0009970 \\
0.0009970 \\
0.0009970 \\
0.9970090
\end{array}\right]
$$

We compute $\mathcal{R}_{0}$ for the new situation

$$
\mathcal{R}_{0}=\frac{\sum_{i=1}^{N} p_{i} a_{i} K_{i}}{c}=10.003327
$$

We compute the new $\frac{r_{i}}{a_{i}}$

$$
(250.75,1003 ., 501.5,0.3343333)
$$

and

$$
\mathcal{F}^{*}=\frac{\sum_{i=1}^{4} p_{i} a_{i} K_{i}-c}{\sum_{i \in J} \frac{p_{i} a_{i}^{2} K_{i}}{r_{i}}}=0.3016127<\min _{i} \frac{r_{i}}{a_{i}}=0.3343333
$$

We have a SFE in the positive orthant.

We remark, that suppressing the two edges comes down to lower the fishing effort on patch 1 and 2 . 


\section{Discussion}

We have developed a multisite fishery model and considered a reduced aggregated model, which approximates the original model. We completely solve the asymptotic behaviour of the aggregated model. Inspired by demography and epidemiology we introduce a basic reproduction ratio $\mathcal{R}_{0}$ for a fishery model. This computable ratio $\mathcal{R}_{0}$ acts as a threshold. We describe the behaviour of the fishery model when the initial condition is in the positive orthant or in the boundary of the nonnegative orthant as well.

In particular when $\mathcal{R}_{0}>1$ the fishery stabilizes to sustainable equilibrium (SFE). We suppose naturally that at the start of the fishing exploitation all the patches are stocked with fish. Otherwise the patch will not be visited by the fishing fleet.

Even if we prove, that when $\mathcal{R}_{0}>1$ the fishery stabilizes to a SFE, this equilibrium is significant, only when it belongs to the positive orthant. Indeed if the SFE is in a face of the orthant, the patches without stock will end to be avoided by the fleet, and then the Coates graph of the fishery will be reconfigured. Consequently the matrix $A$, that we defined in section 1 is modified. This means that the systems (1) and (5) are changed and a new computation of the thresholds must be done. A better strategy will be to act directly on the parameters of the models to ensure that

$$
\sum_{i=1}^{N} p_{i} a_{i} K_{i}>c>\sum_{i=2}^{N} p_{i} a_{i} K_{i}\left(1-\frac{r_{1}}{a_{1}} \frac{a_{i}}{r_{i}}\right) .
$$

is satisfied.

The parameters $r_{i}, K_{i}, p_{i}$ and $q_{i}$ cannot be modified by a strategic modification of the fishery. An exception can be the modification of the species exploited, then leading to a modification of the $q_{i}$.

Another possibility is to modify the Coates graph, or in other words to modify the planning of the fishing season for the fishery. This action will change the $v_{i}$, i.e., the components of the Perron-Frobenius vector of the Coates matrix $A$, for a given total fishing effort. We have shown, in section 5.2 , that by an appropriate change of planning, it is possible to move the equilibrium in the positive orthant, or in other words, does not exhaust any patch. In such a situation it is necessary to explore numerically the different possible Coates graphs, for a given geographical set of patches, and the value obtained by the equilibria. We will pursue this task elsewhere.

\section{References}

J. Arino. Modeling and Dynamics of Infectious Diseases, volume 11 of Contemp. Appl. Math., chapter Diseases in metapopulation models, pages 65123. World Scientific, 2009. 
P. Auger, R. Bravo de la Parra, J.-C. Poggiale, E. Sánchez, and T. NguyenHuu. Aggregation of variables and applications to population dynamics. In Structured population models in biology and epidemiology, volume 1936 of Lecture Notes in Math., pages 209-263. Springer, Berlin, 2008a.

P. Auger, R. Bravo de la Parra, J.-C. Poggiale, E. Sánchez, and L. Sanz. Aggregation methods in dynamical systems and applications in population and community dynamics. Phys. Life Rev., 5:79-105, 2008b.

P. Auger, C. Lett, A. Moussaoui, and S. Pioch. Optimal number of sites in artificial pelagic multisite fisheries. Can. J. Fish. Aquat. Sci., 67:296-303, 2010.

Jørgen Bang-Jensen and Gregory Gutin. Digraphs. Springer Monographs in Mathematics. Springer-Verlag London Ltd., London, second edition, 2009. ISBN 978-1-84800-997-4. doi: 10.1007/978-1-84800-998-1. URL http://dx.doi.org/10.1007/978-1-84800-998-1. Theory, algorithms and applications.

E. Beretta and Y. Takeuchi. Global stability of Lotka-Volterra diffusion models with continuous time delay. SIAM J. Appl. Math., 48:627-651, 1988.

Abraham Berman and Robert J. Plemmons. Nonnegative matrices in the mathematical sciences, volume 9 of Classics in Applied Mathematics. Society for Industrial and Applied Mathematics (SIAM), Philadelphia, PA, 1994. ISBN 0-89871-321-8. Revised reprint of the 1979 original.

J.A. Bonsack, D.L. Johnson, and R.F. Ambrose. Artificial Habitats for Marine and Freshwater Fisheries., chapter Ecology of Artificial Reef Habitat and Fishes., pages 61-107. Academic Press, San Diego., 1991.

W. Chen. Applied Graph Theory; Graphs and Electrical Networks. NorthHolland, 1976.

Colin W. Clark. Mathematical bioeconomics: the optimal management of renewable resources. Wiley-Interscience [John Wiley \& Sons], New York, 1976. Pure and Applied Mathematics.

Colin W. Clark. Bioeconomic modelling and fisheries management. A WileyInterscience Publication. John Wiley \& Sons Inc., New York, 1985. ISBN 0-471-87394-2.

Colin W. Clark. Mathematical bioeconomics. Pure and Applied Mathematics (New York). John Wiley \& Sons Inc., New York, second edition, 1990. ISBN 0-471-50883-7. The optimal management of renewable resources, With a contribution by Gordon Munro, A Wiley-Interscience Publication.

J. Claudet and D. Pelletier. Marine protected areas and artificial reefs : A review of the interactions between management and scientific studies. Aquat. Living Resourc., 17(2):129-138, 2004.

C.L. Coates. Flow graph solutions of linear algebraic equations. IRE Trans. Circuit Theor., CT-6:170-187, 1959.

Tomás de Camino-Beck, Mark A. Lewis, and P. van den Driessche. A graph-theoretic method for the basic reproduction number in continuous time epidemiological models. J. Math. Biol., 59(4):503-516, 2009. ISSN 0303-6812. doi: 10.1007/s00285-008-0240-9. URL http://dx.doi.org/10.1007/s00285-008-0240-9. 
T. Dempster and M. Taquet. Fish aggregation device (FAD) research: gaps in current knowledge and future directions for ecological studies. Rev. Fish Biol., 14(1):253-257, 2004.

O. Diekmann, J. A. P. Heesterbeek, and J. A. J. Metz. On the definition and the computation of the basic reproduction ratio $R_{0}$ in models for infectious diseases in heterogeneous populations. J. Math. Biol., 28(4):365-382, 1990. ISSN 0303-6812.

Odo Diekmann and J. A. P. Heesterbeek. Mathematical epidemiology of infectious diseases. Wiley Series in Mathematical and Computational Biology. John Wiley \& Sons Ltd., Chichester, 2000. ISBN 0-471-49241-8. Model building, analysis and interpretation.

M. Doob. Applications of graph theory in linear algebra. Math. Magazine, 57 (2):67-76, 1984.

S.D. Fretwell and Jr H.L. Lucas. On territorial behavior and other factors influencing habitat distribution in birds. i. theoretical development. Acta Biotheorica, 19:16-36, 1970.

B. S. Goh. Global stability in many-species systems. Amer.Naturalist, 11: 135-143, 1977.

G.D. Grossman, G.P. Jones, and W.J. Seaman Jr. Do Artificial Reefs Increase Regional Fish Production ? A Review of Existing Data. Fisheries, 22(4): 17-23, 1997.

J.K. Hale. Ordinary differential equations. Krieger, 1980.

I. Hanski. Metapopulation Ecology. Oxford University Press, 1999.

I. Hanski and M. Gilpin. Metapopulation Biology, Ecology, Genetics and Evolution. Academic Press, New-York, 1997.

Frank Harary. The determinant of the adjacency matrix of a graph. SIAM Rev., 4:202-210, 1962. ISSN 0036-1445.

Gary W. Harrison. Global stability of predator-prey interactions. J. Math. Biol., 8:159-171, 1979.

J. A. P. Heesterbeek. A brief history of $R_{0}$ and a recipe for its calculation. Acta Biotheorica, 50:189-204, 2002.

Josef Hofbauer and Karl Sigmund. Evolutionary games and population dynamics. Cambridge University Press, Cambridge, 1998. ISBN 0-521-62365-0; 0-521-62570-X.

James E. Humphreys. Linear algebraic groups. Springer-Verlag, New York, 1975. Graduate Texts in Mathematics, No. 21.

Y. Iwasa, S. A. Levin, and V. Andreasen. Aggregation in model ecosystems. I. Perfect aggregation. Ecol. Model., 37:287-302, 1987.

Yoh Iwasa, Simon A. Levin, and Viggo Andreasen. Aggregation in model ecosystems. II. Approximate aggregation. IMA J. Math. Appl. Med. Biol., 6(1):1-23, 1989. ISSN 0265-0746.

J. A. Jacquez and C. P. Simon. Qualitative theory of compartmental systems. SIAM Rev., 35(1):43-79, 1993. ISSN 0036-1445.

J. P. LaSalle. The stability of dynamical systems. Society for Industrial and Applied Mathematics, Philadelphia, Pa., 1976. With an appendix: "Limiting equations and stability of nonautonomous ordinary differential equations" 
by Z. Artstein, Regional Conference Series in Applied Mathematics.

David G. Luenberger. Introduction to dynamic systems. Theory, models, and applications. John Wiley \& Sons Ltd., 1979.

S. Mason and H. Zimmermann. Electronic Circuits, Signals and Systems. Wiley-Interscience, 1960.

R. Mchich, P. Auger, R. Bravo de la Parra, and N. Raissi. Dynamics of a fishery on two fishing zones with a fish dependent migrations : aggregation and control. Ecol. Model., 158(1-2):51-62, 2002.

R. Mchich, N Charouki, P. Auger, N Raissi, and O Ettahiri. Optimal spatial distribution of the fishing effort in a multi-fishing zone model. Ecol. Model., 197:274-280, 2006.

J. Michalski, J.-C. Poggiale, R. Arditi, and P. Auger. Macroscopic dynamic effects of migrations in patchy predator-prey systems. J. Theoret. Biol., 185: 459-474, 1997.

G. Moreno, L. Dagorn, G. Sancho, and D. Itano. Fish behaviour from fishers' knowledge: the case study of tropical tuna around drifting fish aggregating devices (DFADs). Can. J. Fish. Aquat. Sci., 64(11):1517-1528, 2007.

O Ovaskainen and I Hanski. Spatially structured metapopulation models: global and local assessment of metapopulation capacity. Theor Popul Biol, 60:281-302, 2001.

J.R Rooker, Q.R. Dokken, C.V. Pattengill, and G.J. Holt. Fish assemblages on artificial and natural reefs in the flower garden banks national marine sanctuary, u.s.a. Coral Reefs, 16:83-92, 1997.

H.L. Smith. Monotone dynamical systems. An introduction to the theory of competitive and cooperative systems. AMS, Providence, R.I., 1995.

V.L. Smith. Economics of production from natural resources. Amer. Econom. Rev., 58(409-431):3, 1968.

V.L. Smith. On models of commercial fishing. J. Politic. Econ., 77(181-198), 1969.

Y Takeuchi and N. Adachi. The existence of globally stable equilibria of ecosystems of the generalized volterra type. J. Math. Biol., 10:401-415, 1980.

P. van den Driessche and J. Watmough. reproduction numbers and subthreshold endemic equilibria for compartmental models of disease transmission. Math. Biosci., 180:29-48, 2002.

$\mathrm{V}$ Volterra. Leçons sur la théorie mathématique de la lutte pour la vie, volume VII of Cahiers scientifiques. Gauthier-Villars, 1931.

\section{Appendix}

This code computes different value linked to the existence of SFE.

We suppose to have parameters of the "vectorialized" system (2) given in the Scilab command window : namely $\mathbf{r}, \mathbf{K}, \mathbf{q}, \mathbf{p}, \mathbf{c}$. 
In this code we denote by Cost the real $c$ defined by $c=\sum_{i} c_{i} v_{i}$., where $v$ is the normalized Perron-Frobenius vector of $A$.

This code, in a first step, orders the coordinates such that the inequalities (9) are satisfied. Then $\operatorname{rr} 0$, which is $c\left(\mathcal{R}_{0}-1\right)$, is computed. We have to check rro $>0$, a necessary condition for the existence of a SFE.

The second inequality, given by relation (18) is critSFE1 which must be positive. The value of critSFE2, which corresponds to the criteria for the next face in the flag of faces.

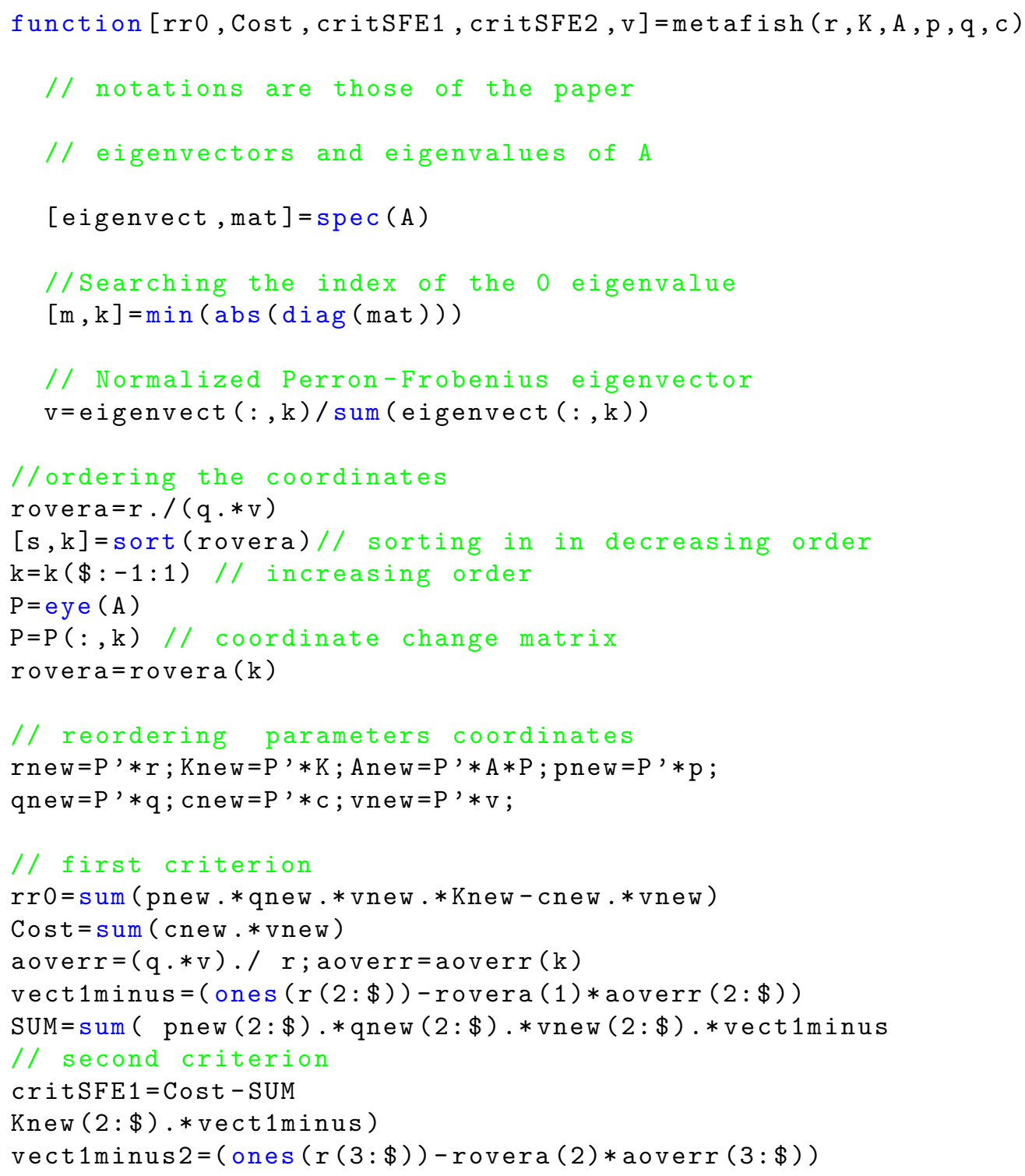


38 SUM2 $=\operatorname{sum}(\operatorname{pnew}(3: \$) . *$ qnew $(3: \$) . *$ vnew $(3: \$) . *$ vect $1 \mathrm{minus} 2$

39 //criterion for next face

40 critsFE2 $=$ Cost - SUM2

$41 \mathrm{Knew}(3: \$)$.*vect $1 \mathrm{minus} 2)$

42 endfunction 